\title{
ENTRE LA RELIGION ET LA LIBERTÉ MODERNE, ENTRE LE SUBI ET LE VOULU: CONSIDÉRATIONS À PARTIR DE LA PENSÉE DE MARCEL GAUCHET ET CELLE DE PIERRE MANENT
}

ENTRE A RELIGIÃO E A LIBERDADE MODERNA, ENTRE O SUPORTADO E O DESEJADO: CONSIDERAÇÓES A PARTIR DOS PENSAMENTOS DE MARCEL GAUCHET E DE PIERRE MANENT

Eduardo Tergolina Teixeira

Defensor Público Federal

Doutorando em Filosofia pela Unisinos e pela Université du Québec à Trois-Rivières eduardotergolina@gmail.com

\section{RÉSUMÉ}

Le présent travail a pour but d'exposer de brèves remarques sur deux éminents philosophes français contemporains : Marcel Gauchet et Pierre Manent. D'abord, je vais exposer la pensée de Gauchet, en soulignant la thèse (qui est présentée surtout dans l'œuvre Le Désenchantement $d u$ monde) selon laquelle la société moderne se trouve actuellement dans un processus inexorable de sortie de la religion. Ensuite, quelques convictions de Manent seront explicitées en ce qui concerne (1) la religion dans la société moderne ; (2) l'influence de la démocratie moderne dans la relation tensionnelle entre la religion et la république et (3) à partir de cela, la question qui se pose quant à la relation entre les trois concepts suivants : vérité, liberté et propriété. Ces trois points seront travaillés à partir du texte Le vecteur théologico-politique, qu'on trouve dans l'œuvre Cours familier de philosophie politique. Finalement, la conclusion sera destinée à mettre en évidence quelques consonances et aussi quelques différences que j'ai trouvées à partir de la lecture de ces penseurs si profonds et renommés.

Mots-clés : Religion. État. Démocratie. Vérité. Liberté. Propriété. 


\section{RESUMO}

O presente trabalho tem por objetivo expor breves notas sobre dois eminentes filósofos franceses contemporâneos: Marcel Gauchet e Pierre Manent. Primeiramente, apresentarse-á o pensamento de Gauchet, sublinhando-se a tese (exposta sobretudo na obra Le Désenchantement du monde) segundo a qual a sociedade moderna encontra-se atualmente em um processo inexorável de saída da religiâo. Em seguida, algumas convicçóes de Manent serão explicitadas no que concerne (1) à religiáo na sociedade moderna; (2) à influência da democracia moderna na relação tensional entre a religião e a república e, a partir daí (3) à questão que se coloca quanto à relaçấo entre três conceitos: verdade, liberdade e propriedade. Estes três pontos serão trabalhados fundamentalmente a partir do texto $L e$ vecteur théologico-politique, encontrado na obra Cours familier de philosophie politique. Finalmente, a conclusão será destinada a colocar em evidência algumas consonâncias e também algumas diferenças encontradas a partir da leitura destes pensadores tão profundos quanto renomados.

Palavras-chaves: Religiāo. Estado. Democracia Verdade. Liberdade. Propriedade.

Data de submissão: 22/03/2018

Data de aceitação: 23/05/2018

\section{MARCEL GAUCHET ET LE PROCESSUS DE LA SORTIE DE LA RELIGION}

D'abord, il est important de noter que Marcel Gauchet, dans son œuvre Le Désenchantement du monde, fait une histoire "politique " de la religion. Il s'intéresse donc au politique. Et, bien qu'il soit un non-croyant, il donne une place très spéciale à la religion dans ce processus de développement historique de la politique dans l'humanité (jusqu’à nos jours).

La période dans laquelle le philosophe français écrit Le Désenchantement du monde, est définie par la fin du marxisme, et Gauchet n'a jamais été marxiste, il s'insère à vrai dire dans une ligne contraire au marxisme. Qu'est-ce qu'est la religion pour le marxisme ? Le marxisme voit dans la religion une forme d'aliénation, une idéologie, une fausse conscience, quelque chose qui ne correspond pas à la réalité. Pour cette théorie, la réalité 
est dans l'histoire réelle, matérielle, non pas dans la " fantasmagorie religieuse »; la religion vient de l'infrastructure, il s'agit d'une mystification, elle est " l'opium du peuple "; la religion est une légitimation de la domination, un facteur d'influence, elle amène le pauvre à accepter sa condition par le moyen de la création d'un autre monde : "si vous n'êtes pas bien ici, si vous n'êtes pas content ici, alors, ne vous inquiétez pas, il y a un autre monde, et vous serez heureux là-bas, après cette vie ici ». Marx considère que la religion, aussi bien que l'État, n'a pas de réalité, elle est une production de la classe dominante et elle va disparaître un jour. ${ }^{1}$

Dans les années 1970, on croyait que la religion était en train de disparaître. On vivait un processus de sécularisation, de sortie de la religion, on ne lui donnait pas beaucoup d'importance. La religion était une illusion, une mystification qui allait s'évanouir. Toutefois, autant on sortait de la religion, autant la religion devenait plus intéressante intellectuellement et un thème privilégié de la pensée. À propos de cela, Gauchet dit:

Paradoxe : depuis deux siècles, la religion n'a cessé de perdre en poids relatifs dans la vie de nos sociétés ; dans le même temps, elle n’a cessé de gagner en importance et en profondeur, aux yeux de ses théoriciens, quant à sa fonction au sein des sociétés. Plus nous nous dégageons de son emprise, plus elle nous paraît rétrospectivement avoir été au cœur du dispositif collectif. ${ }^{2}$

La question qui se pose à partir de cela est : pourquoi la religion est devenue si importante dans l'histoire de la société ? Pourquoi les sociétés sont-elles fondées sur la religion ? Il y avait l'explication marxiste, mais on commençait à remettre en question cette explication. C'est dans le champ de ces interrogations et en fonction de la conviction de ce que la religion "donne un sens » que la recherche de Marcel Gauchet va se développer. Pour Marcel Gauchet, nous sommes dans un processus inexorable de "sortie de la religion ". La société moderne est celle dans laquelle ce long processus de sortie de la religion a gagné force et accélération. Dans l'intelligence du philosophe, afin de pouvoir comprendre ce processus, il n'est pas simplement nécessaire de s'opposer à des théories qui cherchent à renouveler une apologie de la foi et à relativiser les liens et à atténuer l'emprise sur l'homo

$1 \quad$ L'ironie est que le marxisme appliqué dans la pratique, va se démontrer, de part en part, une religion (terrestre). Le marxisme, bien qu'il critique cette "invention " d'un autre monde, finit par apporter cet autre monde à la réalité de l'immanence, en produisant une relation entièrement terrestre avec cet "audelà " et en établissant une logique horizontale au lieu de verticale. Cette conception est manœuvrée par le moyen du concept d'histoire : l'histoire a une fin, qui est cet autre monde (terrestre), la disparition des classes dans la société, et cette fin donne un sens à l'histoire. Il y a, ainsi, une finalité, une cible qui doit être poursuivie, la société sans classes se pose comme un ciel dans la terre.

2 GAUCHET, M.. La démocratie contre elle-même. Paris : Gallimard, 2002, p. 27. 
religiosus de ce qui l'entoure. Il est nécessaire aussi et surtout de prendre garde à l'illusion que nous procure l'athéisme en ce qui concerne la pensée qui soutient que la religion n'est rien de plus qu'une aliénation, une fantasmagorie, une mystification, une chose absolument déconnectée de la réalité sociale et historique. L'auteur du Désenchantement du monde enseigne ainsi :

L'hypothèque à lever est donc double. Il ne suffit pas de s'inscrire en faux contre la reconstruction apologétique dictée par les intérêts de la foi, qui, pour sauver la perpétuité transhistorique de l'homo religiosus relativise autant que faire se peut ses attaches et sa dépendance envers le contexte. Il faut tout autant se déprendre de l'illusion où l'athéisme nous plonge, en nous donnant spontanément à penser que le vrai de la dynamique collective se joue forcément à part et au-dessous de ces émanations fantasmagoriques, qui en disent long peut-être sur la psychologie profonde de l'espèce humaine, ou sur le fonctionnement sauvage de la pensée, mais fort peu sur la nature du lien social et sur le moteur réel de l'histoire. L'écran tendu par ce postulat d'inconsistance n'est pas moins opaque que le premier. Peut-être même nous cache-t-il plus sûrement encore ce que c'est qu'une société structurée par la religion et ce que c'est que la religion considérée en fonction de son point primordial d'application dans la réalité. ${ }^{3}$

Afin de comprendre ces affirmations, il est important de souligner la distinction faite par Gauchet entre la religion et la croyance. Selon le philosophe, l'homme moderne n'a pas cessé de croire. Cependant, le sens de cette croyance va se modifier profondément, comme on va voir dans l'analyse suivante sur Pierre Manent. On ne croit plus à une religion organisatrice de la politique de la société. La croyance est devenue aujourd'hui une question intime, particulière, intérieure à chaque individu. En ce sens, la société moderne - même si elle est constituée de personnes qui croient - est une société dans laquelle la religion n'organise plus, n'oriente plus, ne définit pas les pratiques et les destins politiques. Ainsi, en dépit du fait qu'il y a toujours des croyants (les personnes qui croient dans leur cœur, dans leur âme, intérieurement), la société moderne n’est plus gouvernée par la religion. Cette pensée va être durement critiquée par Paul Valadier, qui accusera Gauchet d'avoir oublié, dans son analyse, le cas des États-Unis, une société moderne dans laquelle - malgré tout le développement, tout le progrès qu'il y a là-bas - la religion est quand même vivante. Gauchet va répondre à la critique de la façon suivante :

Mais c'est très exactement à eux [les États-Unis] que je pensais en

3 GAUCHET, M. Le Désenchantement du monde. Une histoire politique de la religion. Paris : Gallimard. Collection Folio/Essais, 2005, p. 14-15. 
parlant d'une société qui 'comporte une majorité de croyants, mais que la croyance n'organise pas'. Il faut s'entendre, évidemment, sur ce qu'organiser veut dire. Je ne veux pas nier par là le rôle sociologique immense de la religion. Ce que j'ai en vue comme organisation se situe à un niveau incomparablement plus profond - formes de pouvoir, formes du lien entre les êtres, formes de l'inscription dans les choses, formes du penser. ${ }^{4}$

Pour Gauchet, la religion, dans son essence, est trouvée dans les sociétés " primitives ». Ces sociétés dites primitives, archaïques, qui vont perdurer jusqu’au XXe siècle, sont appelées par Lévi-Strauss les sociétés "froides ", en opposition aux sociétés "chaudes ", qui sont advenues à partir de l'invention de l'écriture et de l'État et qui font de l'histoire le moteur de leur développement. ${ }^{5}$ Les sociétés archaïques - dites " froides " par Lévi-Strauss -sont les sociétés religieuses dans leur essence, d'après Gauchet. En fonction de cette pensée, qui soutient que la religion, dans son accomplissement maximal, serait dans le principe et que l'histoire de l'humanité démontrerait, dans ce parcours, jusqu'à l'avènement de notre ère moderne, un déclin du religieux, Paul Valadier accusera Marcel Gauchet d'engendrer un hégélianisme inversé ${ }^{6}$ qui montrerait le dépérissement du phénomène religieux autrefois pur. Cela pourrait permettre à Gauchet, selon Valadier, de justifier de façon perspicace sa position "contraire " à la religion (et surtout contraire au christianisme...). Valadier affirme:

Cette identification de l'essence et du primitif permet de poser ensuite une histoire de la dégradation de la forme pure jusqu'à son effacement. Mais comment asseoir cet hégélianisme inversé pour lequel totalité et achèvement sont au principe et non plus au terme final, chez les primitifs et non dans le christianisme ? Par un troisième postulat, à vrai dire central. 3. La religion pure est celle qui montre 'la vérité de son efficience sociale' (p. 38) ; c'est 'le moment de sa domination sans partage' sur le social, marqué par l'opposition au déploiement de la scission. Où a existé une telle domination??

$4 \quad$ GAUCHET, M. La démocratie contre elle-même. Paris : Gallimard, 2002, p. 85.

5 Selon CANTIN, Serge. Aux sources du Désenchantement du monde de Marcel Gauchet. Éléments pour une généalogie. Studies in Religion / Sciences Religieuses. 34/3-4 (2005), p. 502 ; Lévi-Strauss dit ainsi : "Il ne faudrait donc pas distinguer des sociétés 'sans histoire' et des sociétés 'à histoire'. En fait toutes les sociétés humaines ont une histoire, également longue pour chacune puisque cette histoire remonte aux origines de l'espèce. Mais, tandis que les sociétés dites primitives baignent dans un fluide historique auquel elles s'efforcent de demeurer imperméables, nos sociétés intériorisent, si l'on peut dire, l'histoire pour en faire le moteur de leur développement ». CHARBONNIER, Georges. Entretiens avec Claude Lévi-Strauss. Paris : Union générale d'éditions, 1969, p. 45.

7 GAUCHET, M. La démocratie contre elle-même. Paris : Gallimard, 2002, p. 74. 
La profondeur de ce hiatus entre les sociétés " froides " et les sociétés " chaudes " peut être vue, par exemple, dans l'arrivée des Européens en Amérique. Dans le Nouveau Continent, ceux-ci rencontrent des sociétés primitives, des personnes qui ne valorisent pas le progrès, l'histoire, mais au contraire qui valorisent la répétition du même, le mythe, l'immuable, la fidélité au passé. L'historicité est pour ces personnes un déchet, une perdition. Les Européens découvrent ainsi des êtres tellement différents de leur conception historique européenne qu'ils ne peuvent que ne pas reconnaittre ces êtres comme des humains. ${ }^{8}$

Le processus de sortie de la religion, où la société moderne se trouve aujourd'hui de manière très accentuée, est, selon Gauchet, un processus long et qui a eu son commencement il y a environ cinq mille années. L'apparition de l'État, vers 3,000 avant J.-C., coupe l'histoire en deux et elle établit une façon entièrement nouvelle de mettre en relation la société et la religion. Peu à peu "L'Autre religieux » va cesser d'être antérieur et extérieur au domaine humain et il va commencer à pénétrer au-dedans de celui-ci. ${ }^{9}$ La coupure qui, dans les sociétés " froides ", définissait une séparation entre les individus présents et leurs ancêtres, entre le maintenant et le passé qui doit être respecté, entre la personne et un ordre qui lui, doit être révéré et accepté, cette coupure garantissait l'unité de la société et établissait une égalité entre ses intégrants. Cette coupure, en fonction de l'apparition de l'État, finit par être enlevée et par déclencher l'apparition d'une scission qui maintenant se met à l'intérieur de la société même, en établissant les relations de pouvoir et en divisant les hommes entre des gouvernants et des gouvernés. L'État installe ainsi une hiérarchie, les divisions sociales, le pouvoir de quelques-uns qui devront alors commander, au nom de la divinité, les autres. Ce qui surgit, avec l'avènement de l'État, est "un lent mais irrésistible processus d'immanentisation et d'autonomisation du lien social". ${ }^{10}$ À ce moment, les hommes ont commencé peu à peu à sortir de la religion. L'établissement des séparations entre les gouvernants et les gouvernés, des scissions entre ceux qui ordonnent et ceux qui doivent obéir, ce phénomène si propre à l'apparition de l'État est, d'après Gauchet, le principe de ce processus lent et graduel de sortie de la religion. En ce sens Gauchet dit:

La religion sous sa forme la plus pure et la plus systématique est au départ, dans ce monde d'avant l'État, tel que ses quelques aires privilégiées de survivance, de l'Amérique à la Nouvelle-Guinée, nous auront permis de nous former une image concrète - précaire, assurément, grevée d'incertitudes sans remède, mais à jamais boulever-

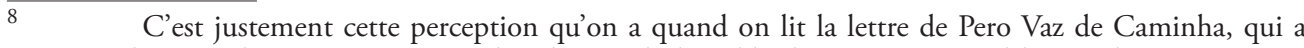
enregistré l'arrivée des portugais au Brésil, in http://objdigital.bn.br/Acervo_Digital/livros_eletronicos/carta. pdf.

$9 \quad$ CANTIN, S. op. cit. p. 501

10 CANTIN, S. op. cit. p. 502.
} 
sante, dans sa fragilité, pour nos quiètes certitudes de civilisés. Dès qu'on se trouve dans l'orbite de la domination institutionnalisée, on est au sein d'un univers où le religieux dans sa radicalité originaire est en question, exposé qu'il est au feu d'une machine à déplacer les horizons de vie, de pensée et d'action dont la dynamique ne cessera plus d'ébranler et de desserrer son emprise. ${ }^{11}$

Il est important de souligner que, chez Gauchet, la religion est essentiellement politique. Et pendant des millénaires, notamment jusqu'à l'avènement de l'État, elle a exercé, avant tout, ce rôle politique. Elle l'a effectué par le moyen d'un régime d'hétéronomie, qui traduit l'idée d'un règlement qui est extérieur à l'organisation des hommes, qui vient du dehors, qui est donné et que les hommes reçoivent (hetero : autre ; nómos : loi). Il y a donc une loi qui vient du dehors, de l'autre, de la divinité. Un tel régime d'hétéronomie englobe l'homme et fait que celui-ci se perçoit en tant qu'une partie du tout qui lui est antérieur, qui est immuable et qui doit être honoré, respecté, obéi et qui ne lui convient pas de questionner. Cette divinité qui agit par le moyen de l'hétéronomie se trouve en intime relation avec le monde de l'immanence et le définit, de telle façon que l'on constate une fusion entre le monde divin et le monde terrestre. Les dieux sont dans le monde, ils agissent dans le monde, le monde est donc enchanté. Il ne convient pas de le dominer, mais de l'accueillir. Et une fois que les dieux sont dans le monde, il n'y a pas de nécessité d'avoir des intermédiaires, des interprètes privilégiés, la relation s'établit directement entre l'homme et le dieu, entre la communauté et la divinité. Les hommes sont une partie d'un tout, ils sont insérés dans un tout, immuable, qui leur est donné et qu'ils doivent accueillir, auquel ils doivent obéir et se soumettre. Il est intéressant de noter que Gauchet perçoit là, dans ces sociétés primitives régies par l'hétéronomie, l'existence d'un choix profondément mystérieux, un " choix arbitraire », fait par les peuples dits archä̈ques. Ce serait comme si, sachant les risques et les dangers inhérents au fait d'assumer leur histoire, ils choisissaient de rejeter leur entrée dans le processus du développement historique, comme si ils " décidaient inconsciemment " de s'arrêter, en se refusant de prendre en charge l'histoire et de devenir autonomes, modernes. Le philosophe français signale que, bien que le processus historique s'impose, peu à peu, qu'il fasse appel à l'homme primitif pour l'intégrer, cet homme - en fonction de ce choix mystérieux et énigmatique - paraît entrer dans l'histoire, mais en se tournant littéralement le dos à luimême. ${ }^{12}$ Ainsi, " demeurer une société essentiellement religieuse ", cela signifierait, en quelque sorte, faire ce choix arbitraire, inconscient, cela serait " opter pour la religion ",

11 GAUCHET, M. Le Désenchantement du monde. Une histoire politique de la religion. Paris : Gallimard. Collection Folio/Essais, 2005, p. 21-22.

12 GAUCHET, M. La démocratie contre elle-même. Paris : Gallimard, Tel, 2002, p. 41. 
pour l'hétéronomie. Gauchet parle d'un homme qui, dans une telle société primitive, choisit de "posséder tout en consentant à sa dépossession ". Et ce serait là, dans ce choix " ambigu ", " paradoxal ", la clé pour comprendre la religion dans sa propre essence. ${ }^{13}$ Paul Valadier va critiquer durement cette thèse de l'existence d'une décision, d'un choix arbitraire pour la religion. Ce penseur soutient que Gauchet, en affirmant cela, donne à comprendre qu'une telle décision pour la religion serait, à vrai dire, une grande équivoque initiale, un péché originel commis par ceux qui ont adhéré au religieux ; cette " option inexplicable " serait une espèce de handicap provisoire, duquel on devrait chercher à se délivrer, Valadier finissant par insinuer que, peut-être, Gauchet aurait pour but, avec cette thèse, de conclure que tous les choix pour le religieux, même les actuels, seraient une façon pour l'homme de refuser d'entrer dans l'histoire et d'agir avec autonomie. ${ }^{14} \mathrm{En}$ réponse à Valadier, Gauchet va affirmer:

\begin{abstract}
Admettre qu'il y ait choix, c'est admettre que dans le principe une autre attitude que religieuse est possible. Il faut donc qu'il n'y ait pas de choix, seule une conception intégrant le sens du sacré comme nécessité constitutive et permanente de l'être humain étant recevable. Cela n'établit pas a contrario la justesse des idées que j'avance. Cela définit simplement leurs conditions de possibilité au plan des présupposés susceptibles de guider ici et maintenant l'appréhension de l'objet religieux, puisque aussi bien c'est sur ce plan que se situent en vérité les objections auxquelles il me faut répondre, et très secondairement sur le plan de la cohérence intrinsèque du propos ou de son adéquation aux faits. [...] Il y a une positivité subjective
\end{abstract}

\footnotetext{
13 "Car ce choix de se posséder en consentant à sa dépossession, en se détournant du dessein de dominer la nature et de légiférer pour son propre compte au profit d'un autre dessein, celui-là de s'assurer d'une identité de part en part définie et maîtrisée, c'est la religion dans son essence même " (GAUCHET, Marcel. Le Désenchantement du monde. Une histoire politique de la religion. Paris : Gallimard. Collection Folio/ Essais, 2005, p. 18).

14 "Mais du coup on aboutit à l'invraisemblable affirmation d'une irrationalité complète, d'une option arbitraire que l'humanité aurait faite de la religion, 'quelque chose comme une décision ou un choix' (p. 33), en réalité une apparence de décision, puisque cet 'acte' s'identifie à une sorte de handicap (provisoire ?) de l'humanité, option inexplicable due à une très remarquable difficulté à assumer ce qui constituait [l'histoire]' (p. 41), une sorte d'entrée (de l'humanité) dans l'histoire 'en se tournant littéralement le dos à elle-même'. Option devant laquelle l'interprète avoue ses 'présentes limites' et son inaptitude à 'maîtriser intellectuellement' ce phénomène. Mais malgré les formules embarrassées, on ne sort pas de l'idée que si l'on ne peut plus expliquer le religieux en le dissolvant dans une structure élémentaire, son existence dans l'histoire relève d'un acte irrationnel et arbitraire : choix qui est une bévue (puisque, le faisant, on tourne le dos à l'histoire) [...] Ici encore ce qui ne se comprend pas soi-même (l'option religieuse qui marque toute l'histoire jusqu’à nous...) est compris par un bon lecteur qui sait, parce qu'il est placé à la fin de l'histoire de la méprise, même s'il avoue ne pas tout savoir : il en sait suffisamment en tout cas pour savoir que le religieux relève d'un péché originel dont il n'est pas dupe, lui du moins. Dire qu'il sait est une façon de parler; il croit savoir plus exactement, grâce à un système de projection de soi qui lui permet de remonter à un acte aberrant comme s'il en était le contemporain. Projection surprenante à moins qu'elle ne soit que le voile mis sur la thèse, plus indécente si elle était affirmée comme telle, que tout choix religieux même actuel est une façon pour l'homme de refuser d'assumer son histoire" (GAUCHET, Marcel. La démocratie contre elle-même. Paris : Gallimard, Tel, 2002, p. 70-71).
} 
de l'expérience du sacré et du rapport de dette à l'invisible dont on peut dégager le noyau primordial et la gamme des transformations. Simplement, dans sa signification individuelle comme dans sa signification collective, l'attitude religieuse me paraît à considérer comme un possible à côté d'autres possibles et non comme une nécessité constitutive et permanente. Et possible hautement sensé - dire que son adoption est sans raison, sans cause extérieure assignable, ce n'est pas dire qu'elle est déraisonnable. Les deux niveaux, personnel et social, communiquent intimement : s'il est une tâche que suggère la théorie de la religion, c'est bien de reconsidérer l'articulation entre organisation subjective et formes du lien social. ${ }^{15}$

Alors, conformément à ce que Gauchet nous enseigne, le processus d'abandon de l'hétéronomie et de sortie de cette logique des sociétés essentiellement religieuse, se déclenche à partir de l'apparition de l'État. Celui-ci établit des séparations dans les organisations sociales, entre les gouvernants et les gouvernés et il internalise dans la société une scission qui n'existait pas auparavant (la division concernait exclusivement le binôme divin-terrestre). Cela provoque un bouleversement inédit dans la structure qui fonctionnait jusqu'à ce moment. En ce sens, l'homme - au lieu de se voir simplement comme une partie d'un tout, comme un élément intégrant, inséré dans un ensemble immuable et qu'il ne peut pas modifier - cherchera à se faire sujet de l'histoire, à transformer ce qui l'entoure, à prendre l'histoire et à en faire le moteur de développement de la société. Commence un processus d'abandon de l'assujettissement, un processus afin de devenir sujet, autonome.

Mais ce processus de sortie de la religion ne sera pas possible sinon à cause de l'occurrence des deux autres discontinuités radicales dans l'histoire de l'humanité, qui suivent l'avènement de l'État. La première discontinuité arrive environ 1,000 avant J.-C. et Gauchet la définira, à partir de Karl Jaspers, la " période axiale ». Cette discontinuité va avoir une atteinte énorme (en Perse, en Chine, en Inde, en Grèce, en Palestine) et elle va consister en un établissement d'une « nouvelle représentation du divin conçu comme radicalement transcendant $" ;^{16}$ cette période est caractérisée par " l'apparition d'une divinité d'outre-monde et d'un rejet religieux de ce monde $"{ }^{17}$ Les religions qui ont surgi à partir de cela (judaïsme, zoroastrisme, bouddhisme, confucianisme, taoïsme et aussi le platonisme), s'insèrent dans un mouvement qui cherche à retirer la divinité du monde de l'immanence, en mettant les dieux dans un plan totalement autre ; ces religions orientent

\footnotetext{
15 GAUCHET, M. La démocratie contre elle-même. Paris : Gallimard, Tel, 2002, p. 87 et 89.

16 CANTIN, S. op. cit. p. 505.

17 GAUCHET, M. Le Désenchantement du monde. Une histoire politique de la religion. Paris : Gallimard. Collection Folio/Essais, 2005, p. 65.
} 
les hommes à "se détacher de leur monde d'apparences et d'illusions " et " créent ce que Nietzsche a appelé des «arrière-mondes» ". ${ }^{18}$ Et Gauchet, en donnant une attention très spéciale au judaïsme, monothéisme (une invention des juifs), va soutenir que, loin de promouvoir une diminution, un aplatissement de l'homme, cette religion finit par le valoriser dans son caractère d'individu, dans la mesure où elle s'appuie sur un Dieu tout transcendent, qui est dans un autre plan, un autre monde, tout divers par rapport au monde terrestre et, à cause de cela, ce Dieu tend ainsi - en fonction de ce détachement de l'organisation sociale - à libérer l'homme. ${ }^{19}$ On dira simplement " tend ", parce que le processus de sortie de la religion et de passage de l'hétéronomie à l'autonomie dépendra encore d'une autre discontinuité radicale : l'avènement du christianisme, qui va permettre le surgissement d'un monde humain autonome à partir du monde religieux. ${ }^{20}$

La nouveauté inexorable du christianisme est justement le phénomène de l'incarnation, un Dieu qui s'incarne, qui se fait homme et qui vient à établir une nouvelle et éternelle alliance entre l'humanité et la divinité. Cela n'avait jamais été conçu auparavant. Mais, en même temps que Dieu s'incarne et habite parmi les hommes, il établit une scission fondamentale entre le monde divin et le monde terrestre. Il révèle que " son royaume n'est pas de ce monde " ${ }^{21}$ comme le dénonce le dialogue célèbre entre Jésus et Pilate. Dans le christianisme, donc, s'instaure le détachement radical entre l'ici-bas et l'au-delà ; Dieu se pose comme un étranger par rapport au monde de l'immanence. Cependant, dans la même mesure où s'annonce cette rupture importante entre le religieux et le terrestre, il y a aussi une valorisation du monde empirique, de l'histoire. Un nouveau monde terrestre s'épanouit, à partir du monde religieux (l'établissement d'une alliance nouvelle et éternelle construit un nouveau monde). L'homme est alors appelé à prendre possession de cette réalité nouvelle, à dominer ce monde et à le transformer. ${ }^{22}$ Le divin se

\footnotetext{
$\overline{18}$ CANTIN, S. op. cit. p. 505.

19 Idem.

20 Ibidem.

21 Jean, $18: 36$. Aussi l'abîme entre les mondes peut être déduit à partir de Luc $16: 26$.

22 Il est important ici de rappeler les enseignements de Descartes : « Mais, sitôt que j’ai eu acquis quelques notions générales touchant la physique, et que, commençant à les éprouver en diverses difficultés particulières, j'ai remarqué jusques où elles peuvent conduire, et combien elles diffèrent des principes dont on s'est servi jusques à présent, j'ai cru que je ne pouvais les tenir cachées sans pécher grandement contre la loi qui nous oblige à procurer autant qu'il est en nous le bien général de tous les hommes : car elles m’ont fait voir qu'il est possible de parvenir à des connaissances qui soient fort utiles à la vie ; et qu'au lieu de cette philosophie spéculative qu'on enseigne dans les écoles, on en peut trouver une pratique, par laquelle, connaissant la force et les actions du feu, de l'eau, de l'air, des astres, des cieux, et de tous les autres corps qui nous environnent, aussi distinctement que nous connaissons les divers métiers de nos artisans, nous les pourrions employer en même façon à tous les usages auxquels ils sont propres, et ainsi nous rendre comme maîtres et possesseurs de la nature " (DESCARTES, René. Discours de la méthode. p. 34-35, disponible in http://bibliothequenumerique.tv5monde.com/livre/33/ Discours-de-la-methode).
} 
détache et le terrestre est livré à l'homme. La relation entre l'homme et le monde temporel est modifiée. L'homme devient sujet et le monde devient objet. Se pose la question qui concerne la domination du monde. Le monde maintenant appartient à l'homme, le terrestre concerne celui-ci, les choses du monde sont livrées à l'être humain, le monde n'est plus animé, enchanté. Il y a maintenant un sujet qui fait ses propres lois, qui prend les rênes du monde, qui le transforme, qui le domine. Le processus de passage de l'hétéronomie à l'autonomie dénonce que la loi maintenant sera délibérée et décidée par l'homme même, par la société même, sans interférences divines, extérieures ; un processus par lequel le monde n'est plus sujet à l'intervention directe et aux commandes exclusives du divin; le divin sort de la scène, il disparaît de l'immanence, dans un processus de désenchantement $d u$ monde. Et dans ce passage de l'hétéronomie à l'autonomie (qui caractérise la société dans laquelle nous vivons aujourd'hui), selon Gauchet, le christianisme joue un rôle capital, étant justement la religion qui a rendu possible cette sortie du monde enchanté, paradoxalement la religion de la sortie de la religion. ${ }^{23}$

D'après Gauchet, la sortie de la religion est un phénomène éminemment occidental. Et, dans le fond, toutes les sociétés sont appelées à sortir de la religion. La rationalité occidentale est imposée partout, et, une fois que nous prenons les outils de cette rationalité moderne, nous sommes amenés inexorablement à entrer dans le processus de sortie de la religion. Même les communautés les plus restreintes, les organisations religieuses les plus ferventes, même les musulmans, par exemple, qui luttent avec beaucoup de vigueur pour résister à ce mouvement, tous - une fois qu'ils prennent les instruments de la modernité - sont insérés à l'intérieur du processus inexorable de la sortie de la religion.

Mais quelles sont les conséquences d'un processus comme celui-ci, de passage de l'hétéronomie à l'autonomie, de sortie de la religion? Selon Gauchet, entrer dans le monde moderne, ce n'est pas entrer dans un monde expliqué par la raison, c'est plutôt entrer dans un monde où la raison tente d'expliquer, sans, toutefois, apporter ces explications souhaitées. Tandis que, dans les sociétés religieuses, il y avait des réponses, des réponses étaient données, la religion apportait des explications; le monde moderne, en revanche, livre la réalité à l'homme, en lui donnant l'autonomie, mais aussi ce monde le laisse à son propre sort. Tandis que l'intégrant de la société primitive n'était pas proprement un individu, mais quelqu'un qui faisait partie d'un tout immuable, lié au passé, à la communauté, il savait quelle était sa place et il recevait des réponses ; nous, modernes, au contraire, nous sommes devenus des individus, sujets, autonomes, entièrement

23 GAUCHET, M. Le Désenchantement du monde. Une histoire politique de la religion. Paris : Gallimard. Collection Folio/Essais, 2005, p. 11. 
responsables, mais aussi dramatiquement dépossédés d'explications. En ce sens, bien que l'autonomie libère l'homme et l'appelle à prendre les rênes de son histoire, à se donner ses propres règles et à transformer le monde, cette autonomie n'apporte pas des réponses aux questions les plus fondamentales de l'existence humaine. Nous sommes des sujets, autonomes, libres, dans une société de démocratie libérale, mais ce n'est pas pour autant que nous avons (nous, les sujets) des éclaircissements à nos questions les plus essentielles. Comme nous indique Alexis de Tocqueville, le passé n'éclaire plus l'avenir et l'esprit maintenant, bien qu'il prenne possession de l'autonomie, marche dans les ténèbres. ${ }^{24}$ Ce risque aurait été détecté par les sociétés primitives, qui donc auraient fait ce choix inconscient de refuser d'entrer dans l'histoire et de devenir des sujets autonomes. Elles possédaient tout en consentant à leur dépossession.

Sortir de la religion, c'est sortir d'un monde régi par une altérité fondamentale, c'est sortir d'un régime de dépossession de soi. L'individu-sujet l'a fait comme cela, quand il a saisi l'autonomie et est entré dans la démocratie moderne. Si, d'un côté, l'homme s'est libéré des entraves propres à l'intégration d'une communauté religieuse, d'un autre côté, la conséquence néfaste qui peut être constatée c'est que, en faisant ainsi, l'homme a fini par s'enfermer dans la production et la transformation du monde, en s'occupant, de façon infatigable et exclusive, à exercer son autonomie ; le résultat de cela est le mépris que l'homme-individu-sujet jette sur la nécessité de réflexion par rapport à toutes les questions les plus fondamentales de sa propre nature ; l'homme moderne finit par escamoter les

«Q "Quoique la révolution qui s'opère dans l'état social, les lois, les idées, les sentiments des hommes, soit encore bien loin d'être terminée, déjà on ne saurait comparer ses œuvres avec rien de ce qui s'est vu précédemment dans le monde. Je remonte de siècle en siècle jusqu’à l'antiquité la plus reculée ; je n'aperçois rien qui ressemble à ce qui est sous mes yeux. Le passé n'éclairant plus l'avenir, l'esprit marche dans les ténèbres ". (TOCQUEVILLE, A. de. De la démocratie en Amérique. Paris : Institut Coppet, 2012, p. 614). 
défis les plus sérieux de son existence même. Les dites " questions sans réponse " ${ }^{25}-$ bien qu'elles viennent parfois inexorablement à troubler la pensée de l'homme moderne finissent par être déconsidérées, méprisées, oubliées.

En ce sens, contrairement à l'homme de la religion, l'homme moderne, autonome, marche dans les ténèbres. Et il est important de noter aussi que, une fois que l'homme moderne est dans l'histoire et qu'il n'a plus la religion pour lui donner des réponses, c'est maintenant la question politique qui va s'imposer et se poser de façon encore plus aiguë. L'homme est seul, autonome, il marche dans les ténèbres de sa rationalité moderne et il doit encore inexorablement chercher à vivre ensemble, avec l'autre, son cosociétaire. Et cela devant une coupure profondément ouverte tout au sein de la société, une coupure qui établit des divisions, des hiérarchies, des pouvoirs et des différences, un grand abîme qui s'est installé comme un héritage à cause de la sortie de la divinité du monde de l'immanence.

\section{PIERRE MANENT. LA LIBERTÉ MODERNE, LAVÉRITÉ ET LA PROPRIÉTÉ}

Dans le Chapitre II (Le vecteur théologico-politique) de son œuvre Cours familier de philosophie politique, Pierre Manent nous enseigne que notre régime de la liberté moderne peut être défini par l'organisation de séparations et il se demande s'il y aurait une séparation

$25 \quad$ Gauchet dit que «Le déclin de la religion se paie en difficulté d'être-soi ». La société d'après la religion est une société " psychiquement épuisante pour les individus, où rien ne les secourt ni ne les appuie plus face à la question qui leur est retournée de toutes parts en permanence : pourquoi moi ? Pourquoi naître maintenant quand personne ne m'attendait ? Que me veut-on ? Que faire de ma vie quand je suis seul à la décider ? Serai-je jamais comme les autres ? Pourquoi est-ce que cela - la maladie, l'accident, l'abandon tombe sur moi ? Â quoi bon avoir vécu si l'on doit disparaître sans laisser de traces, comme si, aux yeux des autres, vous n'aviez pas vécu ? Nous sommes voués à vivre désormais à nu et dans l'angoisse ce qui nous fut plus ou moins épargné depuis le début de l'aventure humaine par la grâce des dieux » (GAUCHET, Marcel. Le Désenchantement du monde. Une histoire politique de la religion. Paris : Gallimard. Collection Folio/Essais, 2005, p. 406). On peut vérifier une similitude remarquable entre ces questions sérieuses que nous pose Gauchet et les Pensées de Blaise Pascal. Quand celui-ci parle de «l'homme placé entre deux infinis ", il affirme que «III - Quand je considère la petite durée de ma vie absorbée dans l'éternité précédant et suivant : le petit espace que je remplis et même que je vois ; abîme dans l'infinie immensité des espaces que j’ignore et qui m'ignorent, je m'effraye et m'étonne de me voir ici plutôt que là ; car il n'y a point de raison pourquoi ici plutôt que là, pourquoi à présent plutôt que lors. Qui m'y a mis ? Par l'ordre et la conduite de qui ce lieu et ce temps a-t-il été destiné à moi ? Memoria hospitis unius diei praetereuntis [L'espoir de l'impie est comme un duvet chassé par le vent, comme l'écume poussée par le flot, comme la fumée qui se perd dans les airs, comme le souvenir de l'hôte d'un jour qui n'a fait que passer]. IV - Pourquoi ma connaissance est-elle bornée ? ma taille? ma durée ? à cent ans plutôt qu’à mille ? Quelle raison a eue la nature de me la donner telle, et de choisir ce nombre plutôt qu'un autre dans l'infinité ? Desquels il n'y a pas plus de raison de choisir l'un que l'autre, rien ne tentant plus que l'autre» (PASCAL, Bl. Les Pensées. Paris : P. Lethielleux, 1896, p. 40). 
qui pourrait être interprétée comme la principale. ${ }^{26} \mathrm{Il}$ mentionne la séparation entre la société et l'État, il note aussi la séparation entre le représenté et le représentant, mais il observe l'existence d'une séparation qui est dotée d'un caractère très spécial, celle qui concerne l'Église, d'un côté, et l'État, de l'autre. Manent affirme que depuis la querelle des Investitures au XIe siècle ${ }^{27}$ jusqu'à la crise en France, déjà au XXe siècle, entre l'Église catholique et la République, l'histoire d'Europe est décisivement marquée par le conflit entre le politique et le religieux, en ajoutant qu'on a le sentiment qu'une résolution minimalement acceptable pour cette question est advenue seulement avec la séparation de l'Église et de l'État.

La République française n'aurait rencontré sa stabilité qu’à partir de la loi de 1905 , Manent argumentant que n'importe quelle tentative de mettre en question cette séparation, cette idée de lä̈cité, ça signifie (en France) de la même manière, mettre en question le régime politique lui-même. La présence islamique croissante dans ce pays aurait ranimé le débat à ce sujet. Une religion où ses membres refusent, ignorent la séparation entre l'Église et l'État, une religion où ses membres croient vraiment et rigoureusement à un Dieu et à son indiscutable intervention dans le champ politique, une religion où les adeptes ne font pas de distinction entre le temporel et le spirituel et qui, parfois, sont prêts à mourir pour cette cause, cela choque et déconcerte.

Aux arguments qui soutiennent que les adeptes de cette religion doivent s'adapter à l'idée de la lä̈cité et qu'ils doivent se soumettre à la séparation religion-État, s'opposent les arguments qui défendent qu'intervenir dans le cadre de la religiosité, en dictant des façons adéquates à sa pratique et à sa manifestation, cela serait attaquer le respect de la propre identité de ces personnes, chose qui ne pouvait être admise dans un régime de liberté moderne. Selon Manent, les défenseurs de ce deuxième courant comparent la question française avec ce qui arrive au Royaume-Uni. Dans ce pays, il y aurait plus de tolérance et les personnes auraient plus de liberté d'expression dans l'espace public pour manifester

\footnotetext{
$26 \quad$ À la page 27 de cette œuvre, Manent écrit ceci sur le thème du mouvement de séparation dans la démocratie moderne : "Si le mouvement de distinction, ou de séparation, est un aspect fondamental de la démocratie moderne, on peut distinguer, ou séparer, plusieurs grandes catégories de séparations. J'en vois au moins six : séparation des professions, ou division du travail ; séparation des pouvoirs ; séparation de l'Église et de l'État ; séparation de la société civile et de l'État ; séparation entre le représenté et le représentant ; séparation des faits et des valeurs, ou de la science et de la vie " (MANENT, P. Cours familier de philosophie politique. Paris : Gallimard, 2007, p. 27).

27

Cette querelle a opposé la papauté et le Saint-Empire romain germanique entre 1075 et 1122 . Tandis que les souverains, détenteurs du pouvoir temporel, comprenaient que le fait de confier des biens matériels aux évêques permettait à ces souverains de choisir les représentants du pouvoir spirituel, le pape, à son tour, soutenait - surtout à partir de la réforme grégorienne - que c'était son devoir de contrôler - et ainsi choisir - les prêtres et les évêques. Ce fait oppose donc le pouvoir temporel et le pouvoir spirituel.
} 
leur croyance sans avoir peur d'être accusées de risquer la séparation religion-État et l'idée de lä̈cité. En ce sens, la laïcité française couperait l'individu en deux, ayant, d'un côté, le croyant (aussi fervent qu'il soit) et, de l'autre, le citoyen, seulement et rien de plus que le citoyen. Les images divulguées dans le journal Le Monde (le 24 août 2016), où des policiers vérifiaient les vêtements d'une musulmane à la plage de Nice, en lui ordonnant qu'elle retire son voile, et ce, devant les gens qui étaient autour d'elle, ces images sont vraiment représentatives du débat sur la laïcité. ${ }^{28}$

Mais au-delà de l'islam, Manent affirme que la relation entre la religion et l'État dans les démocraties modernes ne va pas sans complexités et obstacles. Le penseur présente le problème de l'avortement. Tandis que plusieurs États autorisent l'interruption d'une grossesse non planifiée, non désirée, en argumentant que l'individu dispose de son propre corps et que des problèmes graves peuvent arriver à la femme dans des cas dramatiques comme la situation du viol, l'Église, de l'autre côté, se positionne totalement et radicalement contre ce type de pratique, en soutenant la protection inconditionnelle de la vie dans toutes les circonstances (en ce sens, cette orientation correspond au cinquième commandement du Décalogue qui établit l'interdiction de tuer). Il est intéressant de remarquer les effets d'une telle séparation. Tandis que la femme peut, avec l'appui de l'État, pratiquer l'avortement (au moins dans certaines circonstances ${ }^{29}$ ), le médecin a le droit de refuser de pratiquer cet acte en fonction d'un impératif de conscience. Deux options ressortent, les deux sont protégées par l'État. Il est important de noter que, dans le champ juridique, devant une telle situation de résolution difficile en fonction du conflit entre les principes constitutionnels (l'autonomie, le droit au propre corps, le droit de faire ses propres choix et la préservation de la santé, d'un côté ; la protection à la vie de l'enfant à naître et l'interdiction de tuer, d'un autre côté), le principe de la proportionnalité entre en scène, en appliquant une pondération des biens/intérêts en jeu, en cherchant le moins de dommages possibles selon la situation. En ce sens, des problèmes difficiles comme

28 http://www.lemonde.fr/societe/article/2016/08/24/les-photos-d-une-femme-contrainte-d-enlever-son-voile-a-nice-susci tent-emoi-et-incomprehension_4987497_3224.html

29 Il est utile de mentionner la décision récente (le 29 novembre 2016) de la cour suprême brésilienne qui a délibéré que l'interruption de la grossesse jusqu'au troisième mois ne peut être considérée comme un délit. Ainsi, la pratique de l'avortement, dans ces circonstances, a été décriminalisée. Selon le juge Luís Roberto Barroso, la criminalisation de l'avortement exécuté dans les trois premiers mois de la gestation transgresserait les droits sexuels et reproductifs de la femme, le droit à l'autonomie de faire ses choix et le droit à l'intégrité physique et psychique. Il a dit aussi que cette criminalisation atteindrait surtout les femmes pauvres, obligées d'utiliser le service public de santé et qui n'ont pas de moyen de faire cette procédure de façon privée ou de se déplacer aux lieux où cette pratique est permise et là donc faire l'interruption de la grossesse. En plus, le juge a fait référence que la pratique n'est pas interdite, au moins dans certaines circonstances, aux États-Unis, au Canada, en Allemagne, en France, en Italie, en Portugal, au Royaume-Uni et aux Pays-Bas. (https:// www.stf.jus.br/arquivo/cms/noticiaNoticiaStf/anexo /HC124306LRB.pdf; http://www1.folha.uol.com.br/ cotidiano/2016/11/1836895-aborto-ate-o-terceiro-mes-nao-e-crime-decide-turma-do-suprem o.shtml). 
celui-ci seront, dans la majorité des situations, résolus conformément à des circonstances spécifiques inhérentes à chaque cas particulier.

Cette question se vérifie aussi dans des cas moins graves mais qui également provoquent des débats énergiques. Dans quelques villes aux États-Unis, les crèches de Noël ont été retirées des lieux publics. On a justifié ça par largument selon lequel cette représentation exprime une seule religion, ce qui est contraire à la détermination de la laïcité de l'État. Manent affirme que les critiques à cette opinion, à leur tour, manifestent des préoccupations par rapport aux dangers qu'une séparation totale entre le public et le privé pourrait apporter, l'espace public finissant par, devant une telle laïcité, devenir totalement vide. ${ }^{30}$

Au Brésil (pays inséré dans une culture nettement chrétienne), il y a quelques années, il y a eu un débat très percutant sur la question du retrait des crucifix des départements publics et gouvernementaux. Ce débat juridique a suscité beaucoup de manifestations. Un juge de la cour suprême brésilienne a dit qu'il y avait une certaine exagération de la part du Ministère Public, instance qui a proposé le procès. Ce sujet n'était pas une priorité en comparaison aux autres graves problèmes auxquels l'État brésilien devait faire face (les prisons surchargées, le manque de sécurité dans les rues, les graves violations des droits humains qui ont lieu en dehors et même au-dedans des départements gouvernementaux, comme dans les postes de police). Pour ce juge, beaucoup de signes qui sont vus comme religieux ne seraient, en réalité, que l'expression d'une civilisation occidentale chrétienne. Dans ce sens, la question ne pouvait pas être amenée à des extrêmes. Il dit : «Si on voit cette question en profondeur et si on finit par assumer une position radicale par rapport à ça, je me demande si nous allons finir par réviser le calendrier. Le samedi, le dimanche vont-ils être revus ? Pâques, Noël ? Ce sont des jours fériés nettement religieux. J'espère qu'on n'ordonnera pas de faire tomber la statue du Christ Rédempteur $»^{31}$

Dans le but de chercher à comprendre l'évolution récente du politique et du religieux, chacun en soi-même et aussi dans son interaction l'un avec l'autre, Manent va utiliser

$30 \quad$ Cette pensée peut être constatée aussi dans le « Chapitre VIII - La démocratie sans la nation ? » du livre "Enquête sur la démocratie ", où Manent expose, de façon très percutante, les dangers propres au surgissement d'un vide entre les individus et l'État. Il soutient l'existence aujourd'hui d'une crise du concept de nation. La nation jouait le rôle d'intermédiation entre les individus et l'État. Dans la mesure où cette notion est en train de disparaître, disparaît aussi cette liaison politique entre les individus (maintenant, il y a, d'un côté, le public et, d'un autre, le privé, les individus ; une démocratie sans corps politique, une "démocratie des individus " s'établit, où l'espace politique est devenu complètement vide). In MANENT, P. Enquête sur la démocratie : études de philosophie politique. Paris : Gallimard, 2007,p. 166 et les pages suivantes.

31 http://veja.abril.com.br/blog/reinaldo/geral/gilmar-mendes-critica-discussao-sobre-simbolos-religiosos-em-tribunais-tomara-que-nao-mandem-derrubar-o-cristo-redentor/ 
l'œuvre gauchetienne Le Désenchantement du monde. Conformément à ce qui nous avons vu ailleurs, Gauchet comprend que nous sommes sortis de la religion (religion comprise dans le sens d'organisatrice, structurante de la société). La religion ne commande plus la forme politique de la société, ne définit plus "l'économie du lien social ${ }^{32}$ Et le christianisme est précisément " la religion de la sortie de la religion ». ${ }^{33}$ D'après Gauchet, le problème actuel de la laïcité est inséparable du problème actuel de la religion, le premier étant une conséquence du second.

Le problème actuel de la religion serait l'affaiblissement et la réduction des églises, la perte d'adeptes, la diminution des vocations, mais surtout le problème ne serait pas dans un changement extérieur, quantitatif, mais au niveau qualitatif. Il y aurait une transformation des propres adeptes, qui comprennent la religion d'une façon différente. L'Église ne peut plus définir leurs croyances, orienter leurs choix politiques ou réguler leurs coutumes. Le sens intime de la croyance a changé. ${ }^{34}$ On passe de la tolérance (comprise en tant que principe politique) au pluralisme (où le croyant intègre la vraie possibilité d'existence d'autres croyances légitimes, un pluralisme qui se fait dans la tête des gens). La relativisation intime de la croyance est un produit de notre siècle, l'esprit démocratique pénétrant dans l'intérieur de l'esprit de la foi, en arrivant à la métamorphose des convictions en identités religieuses.

Dans cette conjoncture, Marcel Gauchet - selon Manent - conceptualiserait le croyant contemporain non pas comme celui qui cherche, dans la religion, une vérité objective universelle ou comme celui qui pense comment les hommes devraient croire. Le croyant contemporain est celui qui, en choisissant sa religion, se choisit à lui-même, la croyance se définissant comme une chose subjective, une identité. Il ne cherche plus à convaincre l'autre, à argumenter en sa faveur, à soutenir qui a raison et qui a tort. En revanche, il

\footnotetext{
32 MANENT, P. Cours familier de philosophie politique. Paris : Gallimard, 2007, p. 42.

33 GAUCHET, M. op. cit., p. 11 ; MANENT, P. op. cit., p. 42 ; aussi on peut comprendre cela à partir de GAUCHET, M. La démocratie contre elle-même. Paris : Gallimard, Tel, 2002, p. 55-56 e 84-86 et CANTIN, S. op. cit. p. 504-507.

34 MANENT, P. op. cit., p. 43. Je me rappelle d'un fait qui exprime bien cette situation : en octobre 2005 il y a eu un référendum au Brésil afin de décider d'interdire ou non le commerce d'armes à feu. À la fin d'une messe, le prêtre a fait une orientation politique, en recommandant aux gens de voter en faveur de l'interdiction, en argumentant que les catholiques doivent être un peuple pacifique et doivent lutter ainsi pour un monde de paix. À ce moment-là, j'ai vu deux messieurs qui parlaient sur la question devant la porte d'entrée de l'église et ils disaient que le prêtre ne devait pas se mêler de ce type de sujet et que sa fonction n'est pas celle d'orienter les choix politiques des citoyens. Enfin, l'orientation en faveur de la permission du commerce des armes a gagné $(63,94 \%$ des personnes - 59.109.265 des votes - en faveur du commerce d'armes à feu et $36,06 \%$ de personnes - 33.333.045 des votes - en faveur de l'interdiction). http://www. tse. jus.br/eleicoes/plebiscitos-e-referendos/referendo-2005 ; http://acervo.oglobo.globo.com/em-destaque/ em-2005-63-dos-brasileiros-votam-em-referendo-favor-do-comercio-de-armas-17786376.
} 
n'admet pas la critique, ni l'argumentation contraire à sa croyance. Cela est une question de conviction intime, un choix personnel, une identité. Dans ce sens, la critique adressée contre sa croyance est vue comme un manque de respect, une agression personnelle. ${ }^{35}$

L'absorption de la religion par la démocratie a déterminé la transformation de la République, son grand adversaire. Jusqu'en 1970 (selon Gauchet) jusqu'au Concile Vatican II (d'après Manent), la religion exerçait une hétéronomie collective. Gauchet constate là une institutionnalisation de la vérité objective à laquelle les gens devaient se soumettre. De son côté, la République était une autonomie collective, gagnée en dépit de la religion. Maintenant, la religion est absorbée par la démocratie et la tension disparait. La religion devient une chose individuelle et la République, avec la laïcité, perd son adversaire, son âme, sa compagne. L'indispensable mouvement tensionnel est défait, il s'est évanoui.

Dans ce sens, l'épuisement de la conception républicaine et la ruine du communisme peuvent être interprétés à partir de l'optique suivante : République et communisme sont deux grands projets de construction d'une cité de l'homme, où il fait l'expérience de sa propre souveraineté. ${ }^{36}$ Les deux projets sont retombés. Le totalitaire a échoué, le démocratique a obtenu un succès trop excessif. Manent dit ainsi sur le sujet : «l'autonomie collective la République -, en se dressant contre l'hétéronomie collective - la religion -, a fini par produire le triomphe de l'autonomie individuelle, de la pure démocratie qui a finalement absorbé la République aussi bien que la religion $»^{37}$.

On produit donc, d'un côté, un monde satisfait, dans la mesure où l'individu a obtenu son autonomie complète, et, d'un autre côté, un monde désolé, puisque les questions les plus déterminantes pour l'homme ne sont plus pensées, elles sont ignorées, éloignées de son existence. La réalisation de la démocratie a impliqué le renoncement à penser les problèmes les plus fondamentaux, les plus déterminants de la propre condition humaine. ${ }^{38}$

Manent analyse l'histoire théologico-politique occidentale, dont la singularité est attribuée par Gauchet à la propre singularité de la religion chrétienne, entendue comme « la religion de la sortie de la religion ». En examinant l'histoire des religions, Manent dit

\begin{tabular}{ll}
\hline 35 & MANENT, P. op. cit., p. 44. \\
36 & MANENT, P. op. cit., p. 45. \\
37 & Idem.
\end{tabular}

38 MANENT, P. op. cit., p. 46. Effectivement, Manent et Gauchet sont d'accord en ce point. Nous pouvons nous rappeler dans ce sens de la page 406 du Désenchantement du monde de Gauchet et aussi de la page 40 des Pensées de Pascal, où ces deux grands penseurs posent des questions très sérieuses sur l'existence humaine. 
que les religions païennes, grecque et romaine, sont définies comme des religions civiques, " municipales ", selon Tocqueville. Les dieux sont ceux de la ville, localisés, municipalisés. "La communauté religieuse redouble la communauté politique ${ }^{39}$ Les deux se confondent. À ces religions s'opposent les religions révélées, universalistes, spirituelles, qui transmettent un message de Dieu qui s'adresse à chacun, un Dieu auquel chacun peut se convertir. Dans ce cadre, Manent demande : qu'est-ce qu'il y a de commun entre ces religions révélées et les religions païennes ? Le judaïsme et l'islam certes rejettent l'idolâtrie des religions païennes, mais elles ont en commun avec celles-ci la confusion ou fusion entre le politique et le religieux, même s'il y a des différences : tandis que les dieux grecs sont les dieux de la cité, le peuple d'Israël est le peuple de Dieu. Alors que la religion grecque est moulée sur la forme politique, le peuple d'Israël, en tant que communauté humaine, est encadré sur la forme religieuse, sur la loi conférée par Dieu. L'accueil de la loi de Dieu définit l'appartenance à Israël, on arrive à la même chose dans le cas de l'islam. La loi de Dieu régule toutes les actions des membres de la communauté : la communauté politique est la religieuse, la communauté religieuse est la politique. ${ }^{40}$

Selon Manent, "la religion chrétienne est différente. Elle se définit moins par une loi que par une foi $» .{ }^{41}$ Être chrétien, c'est avant tout croire; croire à certains dogmes, croire à l'incarnation, à la Trinité. Dans ce sens, le christianisme se détourne du monde, en le laissant comme il est, en laissant les institutions politiques telles qu'elles sont, " rendre à César ce qui est à César ". ${ }^{42}$ Alors, on doit "distinguer le domaine de César et celui de Dieu ${ }^{43}$ C'est la raison pour laquelle on attribue au Christianisme, selon Manent, le rôle décisif dans la formation de la démocratie moderne (d'un monde où l'homme est l'auteur souverain de la loi humaine). Manent affirme que, si Gauchet voit le Christianisme en tant que la religion de la sortie de la religion, c'est justement parce que « le Christianisme est la seule religion qui libère l'espace profane ${ }^{44}{ }^{44}$

Mais également le Christianisme n'est pas apolitique. Il établit une " communauté humaine inédite » et pose un problème politique inédit. La communauté humaine inédite est l'Église, une communauté universelle réelle à laquelle tous sont appelés à appartenir, une communauté qui affiche un principe propre, la charité, et une organisation spécifique,

\begin{tabular}{ll}
\hline 39 & MANENT, P. op. cit., p. 46. \\
40 & MANENT, P. op. cit., p. 47. \\
41 & Idem. \\
42 & Matthieu, XXII, 21. \\
43 & MANENT, P. op. cit., p. 48. \\
44 & Idem.
\end{tabular}


une hiérarchie ecclésiastique. Il s'agit d'une communauté non politique, mais qui touche et fait concurrence aux communautés politiques; une concurrence non frontale, directe, mais indirecte, puisqu'elle s'adresse à chaque homme et femme, en leur promettant l'appartenance à une communauté parfaite.

Le problème politique spécifique créé en fonction de la religion chrétienne, c'est l'ambivalence de l'Église. Si, d'un côté, l'Église libèrel'espace profane, laisse les communautés politiques libres de s'organiser (l'Église, au contraire de la synagogue, n'apporte pas une loi politique), de l'autre côté, elle dévalorise les sociétés politiques. Elle critique le principe de ces sociétés (amour de soi et dévalorisation de Dieu) au nom de son principe (amour de Dieu et dévalorisation de soi), en détruisant la légitimité de ces sociétés. ${ }^{45}$ Dans ce cadre, l'Église refuse à la fois de gouverner les hommes, mais dévalorise ceux qui se consacrent à une telle entreprise. Cela ne va finir que par empêcher les hommes de se gouverner d'une manière adéquate. On introduit une confusion qui est dénoncée par beaucoup de grands penseurs, de Marx à Hobbes, de Hobbes à Rousseau, et celui-ci finit par dire que « il a résulté de cette double puissance un perpétuel conflit de juridiction qui a rendu toute bonne politie impossible dans les États chrétiens; et l'on n'a jamais pu venir à bout de savoir auquel du maître ou du prêtre on était obligé d'obéir ».46

Il faut trouver une solution à ce problème. Et la solution sera de couper ce nœud, de mettre en œuvre une grande séparation, de séparer le plus complètement possible le pouvoir de l'opinion (surtout l'opinion religieuse). Il faut priver de fondement la dangereuse et inintelligible notion du pouvoir spirituel. L'institution religieuse n'aura plus de pouvoir, sauf celui d'enseigner à ceux qui veulent l'écouter ; le pouvoir n'aura plus d'opinion, surtout d'opinion religieuse. Manent affirme que cette idée, qui est seulement appliquée au XIXe et XXe siècles, est déjà entièrement conçue au XVIIe siècle.

Mais Manent demande : comment est-ce qu'une institution humaine peut être séparée de toute opinion ? Il faut concevoir qu'indépendamment de leurs opinions sur Dieu, sur le Bien, sur le monde, les personnes désirent, inexorablement vivre, être libres, chercher le bonheur selon leur propre conscience. Il y a, ainsi, des exigences auxquelles l'homme ne peut pas renoncer. Ce sont les droits de l'homme. C'est ici que l'État va trouver son fondement : implantation, protection, garantie des Droits de l'Homme.

\footnotetext{
$45 \quad$ Ibidem.

46 ROSSEAU, J.-J. Du contrat social, ou principes du droit politique. Leipzig: Gerard Fleischer, 1796, p. 300 .
} 
La séparation " pouvoir spirituel » et " pouvoir temporel » cédera sa place à la scission " pouvoir sans opinions " (État laïc) et "opinions sans pouvoir " (société dans sa diversité). C'est dans ces termes qu'est résolue l'énigme posée par le surgissement du christianisme : on établit un État libéral qui protège les droits mis en œuvre dans le domaine de la société civile.

Mais Manent se demande si cette solution est en fait vraiment durable et satisfaisante, en ajoutant que Gauchet suggère que notre formule politique et morale apporte une impasse en ce qui concerne les questions de la vérité et de la communauté. En caractérisant le mouvement politique de la démocratie moderne comme libérateur ou émancipateur, Manent affirme que, au contraire de l'Ancien Régime (où prévalait le mélange entre le pouvoir temporel et celui spirituel et où étaient donnés des mandements inintelligibles), l'Europe et ses colonies de l'Amérique du Nord ont élaboré un pouvoir avec de nouveaux contours, qui ne donne pas d'ordres incompréhensibles, mais un pouvoir qui représente. Il représente les désirs nécessaires, légitimes, en protégeant et assurant les droits à la vie, à la liberté, et à la recherche du bonheur. Il s'agit de l'État représentatif, défini à partir des séparations.

Cette auto-affirmation de la liberté devra passer par la médiation des séparations, séparations qui sont des abstractions. Le citoyen croyant, afin d'être un bon citoyen, doit faire abstraction de sa religion. Et cette auto-affirmation de la liberté s'établit en deux moments : celui de la société et celui de l'État. L'individu est agent dans la société civile et est représenté dans et par l'État. La société civile et l'État sont deux pôles (indispensables) d'un dispositif. ${ }^{47}$

Dans ce sens, les libéraux et leurs adversaires, chacun soutenu par ses propres motifs, auraient raison et seraient devant un même arrière-plan tissé par la liberté moderne. Alors que les libéraux soutiennent que chacun doit pouvoir exercer librement son droit à la vie, à la liberté et à la recherche du bonheur, leurs adversaires défendent que seulement un État puissant et fortement représentatif pourrait être un vrai instrument pour éviter que les pouvoirs sociaux imposent de nouvelles formes d'aliénation et de domination. ${ }^{48}$

Les avancées et les reculs de l'action étatique, les pratiques plus agressives ou les pratiques de retrait de l'intervention de l'État, les régulations et les dérégulations, les oscillations propres à ces mouvements dans la scène politique pourraient être interprétées, d'après

$47 \quad$ MANENT, P.. op. cit., p. 51.

48 Idem. 
Manent, dans une perspective d'auto-affirmation de la liberté. Aussi, le discrédit actuel de l'État et les critiques acheminées vers lui ne révèleraient que le fait que l'État est devenu un instrument, la liberté n'ayant besoin de lui que comme une communauté qui se sert d'un " gardien de square ». Manent dit lumineusement : "L'État le plus moderne serait nécessairement l'État le plus modeste $" .^{49}$ Cet État assume beaucoup plus une fonction spirituelle, symbolique : l'État est nécessaire à la prise de conscience de l'individu comme citoyen. Manent parle d'un " dispositif de la représentation " où l'État serait neutre, abstrait, au-dessus de la société, un État auquel l'individu peut se reporter en tant que citoyen et revendiquer que lui, l'État, le représente. Manent ajoute que cet État se montre indispensable à la réflexion, à la subjectivation de la liberté moderne.

En parlant de la séparation entre l'État et la société, Manent dit que, même ce type de séparation, apporte aussi des effets maléfiques. "Je ne suis jamais tout entier nulle part. Moitié bourgeois, moitié citoyen, je suis toujours inconfortablement et parfois douloureusement divisé $" .^{50}$ Cette critique permet de comprendre des penseurs comme Rousseau (qui a conçu une volonté générale) ou Marx, celui-ci affirmant que, dès lors que l'humanité moderne doit arriver à sa réalisation, il faut que cette séparation entre l'État et la société civile soit dépassée. Manent affirme qu'une telle pensée est compréhensible : un régime qui est basé sur des séparations - et des séparations peut-être artificielles, abstraites -, une situation comme telle déclenchera des mouvements tendant à son abolition ou à son unification.

Dans cette même idée de surpassement de la séparation, on peut entendre le mouvement social et moral décrit au début du texte de Manent ; un dépassement mais sans sortir de la logique de la liberté moderne. Au-delà d'une protection de l'homme sans opinion, de l'homme en général, de l'homme abstrait, on demande maintenant à la société et à l'État la tutelle des droits de l'homme concret, de l'homme avec toutes ses appartenances, ses particularités. ${ }^{51}$ On demande qu'on reconnaisse et qu'on accepte l'identité culturelle, la religion, les orientations, toutes, n'importe lesquelles, et cela à partir de la simple déclaration de l'individu. Un respect inconditionnel est dû à partir du simple moment où l'individu se déclare ainsi. On surmonte le domaine occupé par un pouvoir laïc, éloigné

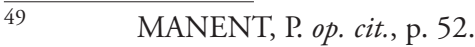

50 Idem.

51 MANENT, P. op. cit., p. 53. Gauchet dit, à son tour, que la politique intéresse l'individu d'aujourd'hui « dans la mesure où elle offre une scène à sa singularité identitaire " (GAUCHET, Marcel. La condition historique. Entretiens avec François Azouvi et Sylvain Piron. Paris, Stock, 2003, p. 329 ; il est intéressant de voir aussi ce que dit Gauchet à la page 334 : "Tout le monde aujourd'hui a quelque chose à demander à la politique, à commencer par la reconnaissance d'une existence ressentie comme tellement précaire qu'elle appelle une certification publique »).
} 
de toutes et quelconques opinions. À sa place rentre un pouvoir qui doit embrasser et accepter énergiquement toutes les opinions et les façons de vivre.

Manent constate l'existence, dans un certain sens, d'un phénomène inversé à celui qui a amené à la séparation entre l'Église et l'État. Pourtant, également, il s'agit d'un prolongement de ce mouvement de séparation, dans la mesure où on n'abandonne pas la logique individualiste et bourgeoise. Et ici Manent accentue le concept de propriétée, ${ }^{52}$ une propriété qui va au-delà des biens, de la vie et de la liberté, une propriété qui atteint les opinions, les valeurs, les identités, les orientations. L'individu est propriétaire de tout ce qui le constitue, et l'État et la société doivent émettre une approbation explicite à ce respect.

A titre de conclusion, Manent affirme que, tout d'abord, on a perçu un ordre qui dépendait d'un pouvoir spirituel, qui cherchait à gouverner seulement de manière indirecte. Afin de surmonter les problèmes qui résultaient de cet ordre (ou désordre), on a envisagé de mettre en œuvre un ordre qui privilégiait l'humain, en ayant ainsi pour but d'établir la séparation entre pouvoir et opinion. Cela constitue la démocratie moderne. Dans l'objectif d'éviter les effets maléfiques de la religion, qui imposait sa vérité, la liberté et la vérité ont été séparées le plus possible. L’homme était défini ainsi par la liberté, on comprenait, après tout, que la vérité restait dans la liberté. Pourtant, la conception de liberté a fini par faire disparaître la vérité. La victoire de la liberté a été excessivement exhaustive. À partir du moment où la relation entre la liberté et la vérité s'effondre, la tension s'évanouissant entre les deux, la liberté perd son adversaire et compagne et tend alors à se ruiner entièrement. La liberté, qui devrait se constituer en un effort vers le gouvernement de soi et par soi (autonomie), finit par être acceptation et déclaration de soi, en exigeant la reconnaissance et l'approbation des autres sociétaires par rapport aux choix faits et aux orientations définies. Ainsi, dans un premier moment, la liberté vainc la vérité, en l'absorbant, mais aussitôt après, cette liberté finit par être vaincue et absorbée par la propriéte..$^{53}$

\section{CONCLUSION}

Le processus de sortie de la religion a amené l'individu moderne au passage de l'hétéronomie à l'autonomie. Dans la démocratie moderne, le sujet autonome revendique la production de ses habilités particulières, la direction de ses affaires privées, la définition de sa propre

\begin{tabular}{ll}
\hline 52 & Idem. \\
53 & MANENT, P.. op. cit., p. 54.
\end{tabular}


vie (puisqu'il est "propriétaire " intégral de celle-ci) ; il n'est donné à personne et à aucune communauté de diriger les choix, les orientations politiques ou personnelles de cet individu. Maintenant, il dicte ses règles et façonne sa vie conformément à ce qui lui plaît. La conséquence d'un tel phénomène est l'isolement qui atteint cet homme moderne, un isolement qui se produit par l'autonomie et qui finit par mouler chacun des individus comme des îles, en leur retirant la conscience d'une participation politique. La notion de communauté commence à leur manquer. La sortie de la religion leur a retiré cette conscience communautaire, de l'être-ensemble. La religion est, avant tout et déjà dans son essence, être en relation avec l'autre. ${ }^{54} \mathrm{La}$ relation hétéronomique avec l'»Autre» se déplace vers la relation avec l'»autre», avec le " prochain ». De plus, l'hétéronomie, dans la même mesure où elle impose une réception des règles à partir de dehors, d'un Autre, établit aussi une relation d'égalité entre les intégrants de la communauté, une absence de divisions, de séparations, ce qui favorise l'être-ensemble. Cette idée nous manque devant l'oubli du paradigme hétéronomique que nous avons fini par laisser en arrière. Et concomitamment à toutes les conquêtes apportées avec l'acquisition de l'autonomie (ces conquêtes que nous, sujets modernes, n'abdiquerions jamais et que nous ne pouvons mépriser), nous sommes, malgré tout, devant l'exigence de vivre ensemble avec l'autre, puisque, bien ou mal, le vivre-ensemble s'impose. En ce sens, si d'un côté le vivre-ensemble est une imposition, parce que le caractère politico-social est le trait distinctif par excellence de l'être humain, d'un autre côté, on a perdu la notion de ce qu'est ce vivre-ensemble, ce vivre en communauté. Avec la sortie de l'hétéronomie et l'assomption de notre autonomie, un problème sérieux nous est imposé, dans un premier plan et d'une façon excessivement claire, à savoir le problème dramatique et énigmatique d'être en rapport avec l'autre, la question de la relation politique.

À partir du parcours que nous avons fait jusqu'à maintenant, nous avons pu constater que les deux philosophes étudiés attribuent, dans des perspectives distinctes, un rôle fondamental au christianisme dans la construction de la démocratie moderne. Gauchet comprend que le christianisme établit, à partir de la conception inédite de l'incarnation, une division claire entre le règne de l'au-delà et celui d'ici-bas et il livre ce monde d'ici à l'homme, qui doit donc le maîtriser. Voilà le pas décisif pour l'entrée inexorable dans le régime de l'autonomie humaine. Le christianisme serait ainsi la religion de la sortie de la religion, et la religion possible pour une humanité d'après la religion (aussi chez Heidegger). ${ }^{55}$ Manent, à son tour, en se référant aux idées d'Hegel, confère un rôle

\footnotetext{
54 GAUCHET, M. La démocratie contre elle-même. Paris : Gallimard, 2002, p. 75.

55 CANTIN, S. op. cit. p. 506.
} 
décisif au christianisme dans le développement historique de l'humanité. ${ }^{56}$ Il critique la conception gauchetienne et affirme que le christianisme ne serait pas la religion de la sortie de la religion, mais que c'est plutôt avec le christianisme que, pour la première fois, la religion apparaît dans son intégrité. ${ }^{57}$

En ce sens, le christianisme est la seule religion qui produit une communauté inédite (l'Église) distincte de toutes les autres communautés politiques. Le chrétien, qui est aussi citoyen, doit effectuer une démarche qui lui est spéciale : d'un côté, il doit être disponible et obéir à un Dieu, le Dieu chrétien, le Dieu de tous les hommes, qui ne fait pas de distinction entre les personnes. D'un autre côté, il doit se gouverner lui-même dans un corps politique distinct, il doit participer à la politique dans un espace de confrontations. Cela serait ce que Manent appelle « l'opération chrétienne ». Et cette opération spéciale va se développer à l'intérieur de la nation. ${ }^{58}$ D'après Manent, cette opération chrétienne est fondamentale pour le développement de la nation, en donnant à celle-ci la force, la consistance, la vitalité, la complexité et aussi les difficultés. Manent affirme ainsi qu’avec la crise du concept de nation et la perte du lien et de la référence chrétienne, l'Europe reste compromise dans son avenir. ${ }^{59}$

Marcel Gauchet et Pierre Manent, deux éminents penseurs contemporains, un athée, l'autre chrétien ; leurs points de vue sont effectivement différents, mais ils ne cessent

\footnotetext{
56 MANENT, P. La nation : forme politique de l'Europe chrétienne. In https://www.youtube.com/ watch? $=0$ s $6 \mathrm{smY} 12 \mathrm{Mhc}$

57 Je pense que Valadier est d'accord avec cela : GAUCHET, M. La démocratie contre elle-même. Paris : Gallimard, 2002, p. 74.

58 Sur le concept de nation, il est intéressant de voir MANENT, P. La nation : forme politique de l'Europe chrétienne. In: https://www.youtube.com/watch?v=0s6smY12Mhc.

59 La critique sur l'évanescence du concept de nation est aussi vue in MANENT, Pierre. Enquête sur la démocratie : études de philosophie politique. Paris : Gallimard, 2007,p. 166 et les pages suivantes. Sur le thème de la nation, Gauchet dit à son tour que les nations étaient construites pour la guerre, mais actuellement on perçoit que peut-être elles sont encore plus profondément construites pour la paix. Parce qu'elles sont faites d'individus, reconnaissent l'indépendance de la société civile et sont faites pour une ouverture les unes sur les autres. En ce sens, les nations admettraient les mêmes principes et une identité de principes ; une nation perçoit qu'il y a d'autres nations autour d'elle et qu'il faut les respecter. Gauchet accentue que, par rapport aux nations, il n'y a plus une vocation de conquêtes, d'élargissement de leurs territoires. L'analyse du concept de nation aujourd'hui doit ainsi être tournée vers la question de la reconnaissance qui doit exister entre les nations. En ce sens, la nature du concept de nation serait ce qui nous permettrait d'envisager un monde pacifié (GAUCHET, Marcel. Entrevue pour Les Rencontres économiques d'Aix-en-Provence, in https://www.youtube.com/watch?v=QvUeWQeggO4). Bien que les deux philosophes perçoivent l'existence d'une crise autour du concept de nation, Manent paraît être plus convaincu de l'évanouissement de cette notion fondamentale.
} 
pas de s'entrecroiser. Gauchet est, si j'ose dire, un optimiste, ${ }^{60}$ peut-être " un optimiste à long terme ", ${ }^{61}$ tandis que la plume de Pierre Manent apporte un fort poids pessimiste. Gauchet est un libéral, ${ }^{62}$ tandis que Manent est un grand critique de l'État libéral moderne. ${ }^{63}$ Les deux perçoivent sérieusement les effets du religieux sur la société moderne et les défis puissants qui se posent devant l'homme qui a gagné son autonomie. Le passage de l'hétéronomie à une autonomie radicale a abouti au surgissement d'un régime où l'homme ne pense qu'à sa liberté individuelle. Dans une "démocratie des individus ", chacun reste isolé, dans sa propre liberté individuelle, chacun soigne ses affaires privées et laisse les questions politiques aux "professionnels de la chose publique ». Apparaît donc un régime politique qui libère les individus du fardeau de la participation politique, libère les individus de penser aux questions de la chose publique. Il y a des bureaucrates professionnels dûment payés pour traiter de telles questions, et donc l'individu est libre de ne penser qu'à ses affaires privées, les siennes et celles de sa famille.

Il apparaît donc une séparation radicale entre le public et le privé : d'un côté, l'espace public mis en marche par des professionnels politiques ; d'un autre côté, les individus qui sont maintenant libres des préoccupations et des agacements de la participation politique et qui peuvent ainsi soigner leurs choses particulières. Un espace vide surgit entre le public et le privé, un espace qui était autrefois justement rempli par la discussion politique, la délibération publique et la participation des citoyens. Maintenant on laisse la politique aux professionnels. Et, libérés du fardeau politique, les individus peuvent ainsi se préoccuper d'eux-mêmes et de leurs affaires. Cela est, d'après Benjamin Constant, la conception de liberté des modernes, en opposition à l'idée de liberté des anciens, pour

\footnotetext{
$\overline{60} \quad$ Gauchet dit ce qui suit, à l'occasion d'une présentation de son œuvre « Comprendre le malheur français ", sur le thème : "Le fait que nous soyons dans une situation très grave ne veut pas dire que cette situation est insoluble. C'est la peine spontanée face à des évènements très lourds qui dépassent notre capacité puis dans un deuxième temps, comme une montagne que se dresse devant vous, on finit par trouver un chemin pour l'escalader. J'aime beaucoup une maxime d'un grand écrivain chinois (..) qui dit à peu près : «le pessimisme ressemble à l'optimisme en ceci qu'il est comme lui une illusion». Ce sont des termes dont il faut donc savoir se dégager. Spontanément, nous sommes pessimistes mais justement l'analyse des raisons qui font que nous sommes pessimistes en général a la vertu de nous donner de l'optimisme. (...) Parce que justement la signification de l'échec - on revient à la question sur le pessimisme et l'optimisme - est de provoquer un violent désir de changement qui finit par trouver son débouché. C'est pour ça qu'en effet il y a un lien dialectique entre le pessimisme et l'optimisme. Les défaites, on les sait dans l'autre sens, n'est-ce pas, on parle de victoire à l'Empire Russe, les victoires où dorment les vainqueurs, et bien il y a des défaites qui réveillent les vaincus et peut-être c'est une situation qui n'est pas sans rappeler ». In https://www.youtube. $\mathrm{com} /$ watch?v=rXMn5a7IjeU.

61 GAUCHET, M. La condition historique. Entretiens avec François Azouvi et Sylvain Piron. Paris, Stock, 2003, p. 325 et 335.

62 Selon CANTIN, S. op. cit. p. 511.

63 MANENT, P. Cours familier de philosophie politique. Paris : Fayard, 2002, p. 52.
} 
lesquels « être libre » signifiait justement participer politiquement aux sujets de la cité. ${ }^{64}$

Mais il est important de rappeler que plus nous nous désintéressons de la politique, plus la politique s'intéresse à nous. Et, si d'un côté la surveillance et le contrôle étatiques sont de plus en plus sophistiqués, d'un autre côté, la relation entre l'individu et ses cosociétaires devient de plus en plus dépourvue de substance. Certes, il n'y a pas de droits absolus et, dans certaines limites, on a la liberté d'aller et venir, de manger et de boire ce qu'on veut, de rencontrer les gens, de profiter des plaisirs et des loisirs. Cependant, dans quelle mesure avons-nous aujourd'hui la liberté pour définir nos priorités en termes politiques ? Dans quelle mesure ne nous trouvons-nous pas au-dedans d'un processus auquel nous ne pouvons rien modifier ou intervenir ? Il est important de nous questionner si aujourd'hui nous faisons effectivement nos propres lois ou si nous laissons les autres s'occuper de notre vie et nous produire intégralement les règles que nous devons suivre et auxquelles nous devons obéir. Il est important de se demander en quoi notre participation politique s'est résumée, et même s'il y a encore réellement une participation politique. ${ }^{65}$ Dans quelle mesure ne sommes-nous pas retournés à être seulement une partie intégrante d'un tout, une partie qui reçoit des lois venues de dehors et qui déterminent, presque en intégralité, notre condition politique ? Dans quelle mesure, dès lors que nous avons conquis notre autonomie, n'avons-nous pas fini par retomber dans une autre espèce d'hétéronomie encore plus radicale, implacable et perverse ? Serait-ce, encore une fois, une tentative de l'être humain de trouver une forme nouvelle d'échapper à l'assomption de sa propre condition d'être sujet, autonome, dans l'histoire ?'66 L'homme serait-il en train de faire à nouveau un " choix mystérieux " afin de fuir l'affrontement des questions les plus cinglantes et insolubles que l'autonomie lui a révélées?

Il y a une différence qui ne peut pas être négligée entre l'homme moderne et l'homme de l'hétéronomie d'autrefois : la situation de l'homme moderne s'aggrave dans la mesure où il a perdu sa référence par rapport au passé, le lien et la conscience ferme d'autrefois sur qui il était. L'homme moderne est un individu, « libre», « autonome», mais ces concepts de liberté et d'autonomie, devant l'absence d'une liaison plus tangible avec le passé et avec les cosociétaires (avec lesquels l'individu est obligé de vivre-ensemble), deviennent chaque

64 CONSTANT, B. De la liberté des anciens comparée à celle des modernes. In http://classiques.uqac. ca/classiques/constant_benjamin/constant_benjamin.html.

65 Les absentions remarquables lors des élections pour choisir les gouvernants sont peut-être un indicatif d'un tel questionnement, encore silencieux, pour la part de la société, celle-ci se demandant sur le sens de cette participation politique dans la démocratie moderne des individus. Gauchet constate qu'effectivement « les populations ne se sentent pas exprimées par leurs représentants » (GAUCHET, M. La condition historique. Entretiens avec François Azouvi et Sylvain Piron. Paris, Stock, 2003, p. 335).

66 GAUCHET, M. La démocratie contre elle-même. Paris : Gallimard, 2002, p. 42. 
jour plus éthérés, graduellement plus difficiles à être définis minimalement. Il s'agit d'un homme qui n’a pas de passé et qui envisage un avenir, qui a perdu la référence et qui vit dans un vide. Cet homme marche dans les ténèbres. ${ }^{67}$

La netteté avec laquelle ces deux éminents penseurs de notre temps tissent leurs diagnostics sur la question politique contemporaine est surprenante et nous déconcerte. À partir de la reconnaissance et de l'assimilation en profondeur des graves difficultés que nous rapportent les thèses manentienne et gauchetienne, «oxalá », nous pouvons nous réveiller - conformément à ce que dit Gauchet -, afin de, en face de cette montagne qui se dresse devant nous, trouver un chemin qui nous permet de l'escalader.

\section{BIBLIOGRAPHIE}

CANTIN, S. «Aux sources du Désenchantement du monde de Marcel Gauchet. Éléments pour une généalogie », Studies in Religion / Sciences Religieuses, 34, 3-4, 2005, p. 495-513.

CHARBONNIER, G. Entretiens avec Claude Lévi-Strauss, Paris, Union générale d'éditions, 1969.

CONSTANT, B. De la liberté des anciens comparée à celle des modernes. In http://classiques. uqac.ca/classiques/constant_benjamin/constant_benjamin.html.

DESCARTES, R. Discours de la méthode. In http://bibliothequenumerique.tv5monde. com/livre/33/ Discours-de-la-methode.

GAUCHET, M. La démocratie contre elle-même, Paris, Gallimard, 2002.

La condition historique. Entretiens avec François Azouvi et Sylvain Piron. Paris, Stock, 2003.

67 À propos, ces ténèbres, vécues par cet homme «libre " gagnent des contours intéressants sous la plume de Manent, quand celui-ci indique que le concept de vérité a succombé à celui de la liberté, ces deux notions finissant par céder leur place au concept de propriété. Effectivement, une telle analyse présente une consonance impeccable avec l'idée de l'individu-sujet-moderne, qui se pose actuellement comme un propriétaire authentique de ses orientations, ses conditions, ses choses, ses identités, il est propriétaire de sa vie. Le concept de propriété s'élargit et assume ainsi une considération et une importance inédites. 
. Le Désenchantement du monde. Une histoire politique de la religion, Paris, Gallimard, 2005.

. Entrevue aux Rencontres économiques d'Aix-en-Provence, page consultée le 15 décembre 2016, https://www.youtube.com/watch?v=QvUeWQeggO4

. Entrevue à la Cité des Livres - la Fondation Jean Jaurès, page consultée le 15 décembre 2016, https://www.youtube.com/watch?v=rXMn5a7ljeU

MANENT, Pierre. Cours familier de philosophie politique, Paris, Gallimard, 2007.

Enquête sur la démocratie : études de philosophie politique, Paris, Gallimard, 2007.

- La nation : forme politique de l'Europe chrétienne, page consultée le 15 décembre 2016, https://www.youtube.com/watch?v=0s6smY12Mhc.

PASCAL, B. Les Pensées, Paris, P. Lethielleux, 1896.

ROUSSEAU, J.-J. Du contrat social, ou principes du drot politique, Leipzig, Gerard Fleischer, 1796.

TOCQUEVILLE, A de. De la démocratie en Amérique, Paris, Institut Coppet, 2012.

Autres pages consultées sur Internet :

CAMINHA, P. V de. Lettre au Roi de Portugal. In: http://objdigital.bn.br/Acervo_Digital/ livros_eletronicos/carta.pdf.

http://www.lemonde.fr/societe/article/2016/08/24/les-photos-d-une-femme-contrainted-enlever-son-voile-a-nic e-suscitent-emoi-et-incomprehension_4987497_3224.html https://www.stf.jus.br/arquivo/cms/noticiaNoticiaStf/anexo/HC124306LRB.pdf 
http://www1.folha.uol.com.br/cotidiano/2016/11/1836895-aborto-ate-o-terceiro-mesnao-e-crime-decide-turma-do-supremo.shtml

http://veja.abril.com.br/blog/reinaldo/geral/gilmar-mendes-critica-discussao-sobresimbolos-religiosos-em-tribunais-tomara-que-nao-mandem-derrubar-o-cristo-redentor/

http://www.tse.jus.br/eleicoes/plebiscitos-e-referendos/referendo-2005

http://acervo.oglobo.globo.com/em-destaque/em-2005-63-dos-brasileiros-votam-emreferendo-favor-do-comercio-de-armas-17786376 